\title{
Microbial Profile of Various Catheter Tips among Hospitalized Patients
}

\author{
Pushpa Man Shrestha ${ }^{1}$, Nisha Thapa ${ }^{2}$, Navraj Dahal ${ }^{3}$, Nabaraj Adhikari ${ }^{4}$, Upendra Thapa Shrestha ${ }^{4 *}$ \\ ${ }^{1}$ Assistant Professor, Ayurved Campus, Institute of Medicine, TU, Kirtipur \\ ${ }^{2}$ Kantipur College of Medical Science, Kathmandu \\ ${ }^{3}$ Senior Medical Microbiologist, B\&B Hospital, Gwarko, Lalitpur, Nepal \\ ${ }^{4}$ Assistant Professor, Central Department of Microbiology, Tribhuvan University, Kirtipur, Kathmandu
}

*Corresponding author: Upendra Thapa Shrestha, Assistant Professor, Central Department of Microbiology, TU, Kirtipur, Kathmandu; E-mail: upendrats@microbiotu.edu.np

\begin{abstract}
Objectives: This study aimed to identify the microbiological profile of various catheter tips, and multidrug resistance pattern of extended spectrum $\beta$-lactamase (ESBL) producing E. coli and Klebsiella spp. isolates.
\end{abstract}

Methods: A descriptive analysis of 263 catheter tip specimens processed for culture and antimicrobial susceptibility testing was carried out in B\&B Hospital, Lalitpur. Five different types of catheter tips were analyzed for microbiological growth and antimicrobial susceptibility testing.

Results: Among catheter tips, the highest percentage of microbial growth was observed in tracheostomy tip. Monomicrobial growth was recorded in $82.9 \%$ catheter tips and polymicrobial growth was observed in $17.1 \%$ tip samples. Of 180 isolates, gram negative rods $(76.6 \%)$ followed by yeast (19.4\%) and gram-positive cocci (3.9\%) were isolated. Gram negative Acinetobacter spp. (25\%) and Pseudomonas spp. (23.3\%) and gram-positive Enterococcus spp. (2.2\%) were the most frequently isolated bacteria. However, carbapenam was the most effective antibiotic for both groups.

Conclusion: Of the total isolates tested, $61.4 \%$ were found to be multidrug resistant (MDR). Among gram negative rods, $22.2 \%$ E. coli and $27.3 \%$ Klebsiella spp. were confirmed as ESBL producer. It is recommended to apply standard protocol during insertion and removal of catheter which may help in managing nosocomial infection associated with catheters.

Key words: Indwelling devices, catheters, nosocomial infection, MDR, ESBL

\section{INTRODUCTION}

Catheter is a tube that can be inserted into a body cavity, duct, or vessel thereby allowing drainage, injection of fluids, or access to surgical instruments. The number of intravascular catheters, urinary catheters, endotracheal tubes and other temporary devices inserted each year probably ranges into the millions. The variety of available devices and the frequency with which they are implanted will undoubtedly continue to increase in the coming years (Dickinson and Bisno 1989).

Due to the frequent and sometimes unnecessary use of indwelling catheters during hospitalization in 21 to

Date of Submission: August 10, 2018

Published Online: January 2019
$50 \%$ of patients, many patients are placed at risk for complications associated with the use of these devices (Jain et al. 1995). A study of 1,540 nursing home residents determined that the risk of hospitalization, length of hospitalization, and length of antibiotic therapy were three times higher in catheterized residents than in non-catheterized residents (Kunin et al. 1992). They are also the main source of bacteremia and septicemia in hospitalized patients (Elliott et al. 1997). Primary blood stream infections (BSIs) comprise the majority (64\%) of nosocomial infections reported by the Centers for Disease Control and Prevention (CDC)'s National Nosocomial Infection Surveillance (NNIS) system, and

Date of Acceptance: November 21, 2018

DOI: https://doi.org/10.3126/tujm.v5i0.22299 
most are due to infected intravascular, mostly central venous catheters. More than 250,000 vascular catheter related bacteremia and fungemia occur annually in the USA with an attributable mortality ranging from $12 \%$ to $25 \%$ in critically ill patients (O'Grady et al. 2002). The prolonged use of intravascular catheters and its improper management is a major risk factor for development of nosocomial BSIs. The worldwide increase in the incidence of nosocomial BSIs is mainly attributed to the increased use of invasive devices and aggressive drug therapy along with increased frequency of invasive procedures (Mermel 2000). Approximately $25 \%$ of central venous catheters (CVCs) inserted have been reported to become colonized with rates of catheter related blood stream infection (CRBSI) varying between $0 \%$ and 11\% (Maki et al. 1997).

It is estimated that $10-12 \%$ of hospital patients and $4 \%$ of patients in the community have urinary catheters in situ at any given time (Stamm and Coutinho 1999). Nosocomial urinary tract infections (UTIs) develop in $5 \%$ of catheterized patients per day in the US, with associated bacteremia in $4 \%$ and as many as $80 \%$ are a consequence of urinary catheters. Fever, pyelonephritis, urinary tract stones and chronic renal inflammation are some of the other complications of this procedure (Sedor and Mulholland 1999).

Bacteria have the capacity to adhere to and multiply on the surfaces of catheters. It may contaminate the system at the insertion site with spread along the external surface of the catheter, in the fluids being infused, or at junctions in the external line. The latter is frequently the result of manipulation of the line by medical personnel. Organisms colonizing CVCs include Coagulase negative Staphylococci (CoNS), Staphylococcus aureus, Pseudomonas aeruginosa, Klebsiella pneumoniae, Enterococcus faecalis, and Candida albicans (Elliott et al. 1997; Raad et al. 2007)

This study is an attempt to summarize the pattern of microorganisms and their resistance pattern that are isolated from the various catheter tips from hospitalized patient in B \& B hospital.

\section{MATERIALS AND METHODS}

This was a cross sectional study carried out at B \& B Hospital (Pvt.) Ltd., Lalitpur, Nepal. During this study, catheter tips were enrolled from both sexes and all age group but catheter tips arrived in saline or transport media, and without proper labeling were excluded.
During this study period, a total of 263 catheter tips samples were collected from hospitalized patients and processed in microbiology laboratory of the hospital. Catheter tips were kept in body for not more than 13 days.

Various tips were removed by nursing staff. The skin was cleaned with $70 \%$ alcohol prior to catheter removal. Observing aseptic technique, the exposed end of catheter was hold and it was removed from the patients with a sterile instrument, taking care to avoid contact with exposed skin. Holding the distal end over a sterile tube, the tip was cut with a sterile scissors, dropping the last 2 to 3 inches into the tube ${ }^{11}$. The tube with tip was labeled with patient name, sample collection date and hospital number. The tube was sealed to avoid the drying and was submitted to Microbiology laboratory as soon as possible.

All the tips were laid on the Blood Agar Plate (BA) and MacConkey Agar Plate (MA). The tip was rolled back and forth exerting a slight downward pressure across the entire surface of a BA and MA using sterile forceps. The plates of BAP were incubated at $35^{\circ} \mathrm{C}$ in $\mathrm{CO}_{2}$. The plates were read at 24, 48, 72, and 96 hours. Each type of colony isolated was counted comparing growth on each medium. Plates were observed for fungal growth.

The identification of various gram-negative isolates was done by using standard microbiological techniques as described in Bergey's Manual of systemic bacteriology and Clinical Microbiology Procedure Handbook (Isenberg 2004).

Antimicrobial susceptibility test towards isolated organisms were performed by Kirby-Bauer disk diffusion method and interpreted according to Clinical Laboratory Standard Institute (CLSI). The initial screen test for the production of ESBL was performed by using both ceftazidime (CAZ) $(30 \mu \mathrm{g})$ and ceftriaxone

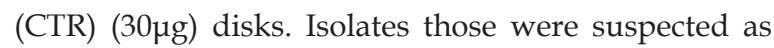
ESBL- producer by screen test were tested further by Combination disk method (CD).

\section{RESULTS}

In this study, a total of 263 catheter tip samples were taken from hospitalized patients at B \& B Hospital, Lalitpur. Out of 263 samples, 152 (57.8\%) were found to be culture positive. Eighty percent of tip culture positive result was observed in a patient re-catheterized for 10 times and $45.7 \%$ tip showed positive culture result from 70 patients with single time of catheterization. 




Types of catheter tips

Figure 1: Growth pattern according to sample distribution

In total 152 culture positive sample, 180 organisms were isolated. Out of 180 organisms, 138(76.6\%) were Gram negative rods followed by 35 (19.4\%) yeast and 7(3.9\%) gram positive cocci. The most predominant organism was Acinetobacter spp. accounting for $25 \%$ of the total isolates.

Table 1: Distribution of various organisms in growth positive tips

\begin{tabular}{lcc}
\hline Organisms & Number & Percentage \\
\hline Bacteria & 145 & 80.5 \\
Gram positive cocci & 7 & 3.9 \\
Enterococcus spp. & 4 & 2.2 \\
MR-CONS & 1 & 0.5 \\
MS-CONS & 1 & 0.5 \\
NHS & 1 & 0.5 \\
Gram negative rods & 138 & 76.6 \\
Acinetobacter spp. & 45 & 25 \\
Pseudomonas spp. & 42 & 23.3 \\
Klebsiella spp. & 22 & 12.2 \\
E. coli. & 21 & 11.7 \\
Proteus spp. & 4 & 2.2 \\
Enterobacter spp. & 3 & 1.7 \\
Citrobacter spp. & 1 & 0.6 \\
Yeast & 35 & 19.4 \\
Candida albicans & 6 & 3.3 \\
Non-albican Candida & 29 & 16.1 \\
\hline
\end{tabular}

Ten different antibiotics were used for all isolates for antibiotic susceptibility test but additional antibiotic colistin sulphate was used for Pseudomonas spp. and Acinetobacter spp. The antibiotic colistin sulphate was found to be $100 \%$ sensitive towards Pseudomonas spp. and $97.8 \%$ Acinetobacter spp. and both of them were resistant to ciprofloxacin, ofloxacin and ceftriazone. Imipenam was found drug of choice for E. coli, Proteus spp., Enterobacter spp. and Citrobacter spp. with sensitivity of $95.4 \%, 100 \%, 100 \%, 100 \%, 100 \%$ of isolates respectively. 
Table 2: Antibiotic susceptibility pattern of Gram-negative rods

\begin{tabular}{cccccccc}
\hline \multirow{2}{*}{$\begin{array}{c}\text { Antibiotics } \\
\text { used }\end{array}$} & $\begin{array}{c}\text { Pcinetobacter } \\
\text { spp. }\end{array}$ & $\begin{array}{c}\text { Pseudomonas } \\
\text { spp. }\end{array}$ & $\begin{array}{c}\text { Klebsiella } \\
\text { spp. }\end{array}$ & $\begin{array}{c}\text { E. } \\
\text { coli }\end{array}$ & $\begin{array}{c}\text { Proteus } \\
\text { spp. }\end{array}$ & $\begin{array}{c}\text { Enterobacter } \\
\text { spp. }\end{array}$ & \multicolumn{2}{c}{ Citrobacter } \\
spp.
\end{tabular}

(Note: Cip- Ciprofloxacin, Of- Ofloxacin, C- Chloramphenicol, Ctr- Ceftriazone, Ptz- Piperacillin/ Tzobactum, Cs- Cefoperazone/ Sulbactum, Imp- Imepenam, Mrp-Meropenam, , Gen-Gentamycin, Ak- Amikacin, CoColistin sulphate)

Out of 7 Gram positive cocci, Enterococcus spp. was found $100 \%$ resistance to six antibiotics. $50 \%$ of CoNS was found resistant to methicillin. Non-haemolytic streptococci (NHS) was found 100\% sensitive towards Chloramphenicol, Amoxycillin and Vancomycin.
Out of 138 Gram negative rods, Citrobacter spp. was found $100 \%$ resistant to $\geq 3$ common antibiotics. While $27.3 \%$ Klebsiella spp. were found to be MDR. Out of 7 Gram positive bacteria, Enterococcus spp. and NHS were found $100 \%$ MDR and $50 \%$ CoNS were MDR.

Table 3: MDR among Gram negative rods

\begin{tabular}{lccc}
\hline Organisms & Total number & MDR (n) & Percentage \\
\hline Acinetobacter spp. & 45 & 43 & 95.5 \\
Pseudomonas spp. & 43 & 20 & 47.6 \\
Klebsiella spp. & 22 & 6 & 27.3 \\
E. coli & 21 & 9 & 42.8 \\
Proteus spp. & 4 & 2 & 50 \\
Enterobacter spp. & 4 & 3 & 75 \\
Citrobacter spp. & 1 & 1 & 100 \\
\hline
\end{tabular}

Table 4: MDR among Gram positive bacteria

\begin{tabular}{lccc}
\hline Organisms & Total & MDR $(\mathbf{n})$ & Percentage \\
\hline Enterococcus spp. & 4 & 3 & 100 \\
CoNS & 2 & 1 & 50 \\
NHS & 1 & 1 & 100 \\
\hline
\end{tabular}

Table 5: ESBL producing E. coli and Klebsiella spp. among Gram negative rods

\begin{tabular}{lcc}
\hline Organisms & ESBL(n) & Percentage \\
\hline E. coli & 2 & 22.2 \\
Klebsiella spp. & 6 & 27.3 \\
\hline
\end{tabular}


Out of 21 E. coli and 22 Klebsiella spp., 22.2\% of E. coli was confirmed to be ESBL producer while 27.3\% Klebsiella spp. was detected as ESBL producer.

\section{DISCUSSION}

This study showed that among 263 catheter tip samples, $152(57.7 \%)$ samples were found to be culture positive. In previous study on growth positive tip culture was found $37.8 \%$ (Atela et al. 1997) and other investigators elsewhere in the world have reported higher growth positive which is in agreement with the findings of the present study (Kalsoom and Abdul 2006; Mahto et al. 2013). Possible reasons for these differences in rates could be the use of central catheters only for very sick patients, absence of dedicated IV catheter insertion teams and lack of standardized protocol for replacement/ change of catheters. The slightly higher growth in our setting may be due to the lack of aseptic technique during insertion and removal of catheter tips, long duration of catheterization and irregular sterilization of patient's room. The study showed that the prevalence of tip colonization increases with the increase in times of catheterization. In this study lowest of $45.7 \%$ growth was observed in tips with single time of catheterization and maximum growth was observed in a patient recatheterized for 10 times with $80 \%$ growth positive results. The catheter tips were kept inside body for maximum 13 days.

In this setting, highest growth was recorded in tip samples received from patients in ICU showing ICU to be more prone to infection. Intensive care unit (ICU) is one of the potential sources of nosocomial infections even in countries where extensive infection control measures are routinely implemented. The international study of infections in ICU, which was conducted in 2007, demonstrated that the patients who had longer ICU stays had higher rates of infection, especially infections due to resistant Staphylococci, Acinetobacter spp., Pseudomonas spp., Candida spp. (Radji et al. 2011).

This study showed a highest growth in tracheostomytip followed by Urinary catheter, Et- tips, Suction tip, CVP and the least growth was in Drain tips. 70.9\% urinary catheters were found to have significant growth in our setting which was comparable with the result of a study done in India in which $69.6 \%$ catheters were found to have microbiological growth (Deep et al. 2004). In our study $66.7 \%$ endotracheal tips were found to be tip culture positive.
Furthermore, in the present study $26(17.1 \%)$ tips to have polymicrobial growth. In the previous studies found that catheter tips with polymicribial growth were $41.2 \%$ and $43.5 \%$ respectively (Storti et al. 2006; $\mathrm{Xu}$ et al. 2012). Risk factors for polymicrobial infections in children and adults include the presence of a central venous catheter, administration of parenteral nutrition, gastrointestinal pathology, especially short gut syndrome, use of broad-spectrum antibiotics and immunosuppression (Downes et al. 2008).

In this study, out of 180 isolated organisms, gram negative bacteria accounted for higher percentage with $76.6 \%$ followed by yeast $19.4 \%$ and gram-positive cocci $3.9 \%$. The high prevalence of gram-negative bacteria may be due to immune compromised state of patients, contaminated infuscate and misuse of antibiotics (Maki et al. 1997; Mermel 2000). The hands of health-care workers often introduce Gram-negative organisms during the manipulation of catheters or intravenous tubing (Gaynes 2009). An intravascular catheter tip colonized with Enterobacteriaceae and Pseudomonas spp. was predictive of subsequent Gram-negative bacteremia in 20 and $14 \%$ of the cases, respectively (Peacock et al. 1998).

Acinetobacter spp. was the most predominant organism accounting for $25 \%$ of total isolates and Pseudomonas spp. was second most prevalent organism. In previous study, Acinetobacter spp. (26.67\%) was most frequently isolated organism followed by Pseudomonas spp. (Tullu et al. 1998). In contrast to our result, it was found that Pseudomonas spp. as most frequent organism followed by Acinetobacter spp. Pseudomonas aeruginosa and Acinetobacter baumannii are the most prevalent nonfermentative bacterial species isolated from clinical specimens of hospitalized patients (Karlowsky et al. 2004). In contrast, CoNS was the most predominant organism in many studies (Riboli et al. 2014).

In the study, yeast was isolated in $19.4 \%$ among all isolates. This result was comparable with the previous study in which prevalence of Candida spp. was 20\% (Rao et al. 2005).

Furthermore, Imipenam was drug of choice for Acinetobacter spp. with efficiency of $80 \%$ and ciprofloxacin, ofloxacin, ceftriazone were found ineffective for the organism. This result was concurrent with previous study showing imipenam as most sensitive drug (75\% sensitive) (Amin, Shrestha and 
Bhat 2013). Amikacin and cefoperazone - sulbactum were effective drug ( $35.7 \%$ each) for the organism in previous study (Khanna et al. 2013). The most effective antibiotic for Pseudomonas spp. was colistin sulphate with $100 \%$ sensitive result followed by meropenam (92.9\% sensitive) and cefoperazone -sulbactum (73.3\% resistant) was ineffective drug in the study.

In addition, overall $61.4 \%$ organisms were found to be MDR which were resistance to three or more than three different group of antibiotics. In previous studies showed that rate of MDR organism was $31.48 \%$ and 30.2\% respectively (Khanna et al. 2013; Parameswaran et al. 2011). The study showed highest number of MDR in Enterococcus spp., Citrobacter spp. and NHS (100\% each) followed by Acinetobacter spp. (95.5\%), Enterobacter spp. (75\%), Proteus spp. and CoNS (50\% each), Pseudomonas spp. (47.6\%), E. coli (42.8\%) and Klebsiella spp. (27.3\%). Isolating MDR A. baumannii from hospitalized patients depends on external ecological variables and risk factors related to the patients themselves (Bonten et al. 1998). Several previous reports have discussed the risk factors associated with the development of MDR A. baumannii infections in hospitalized patients (Allen and Hartman 2004).

In this study $18.6 \%$ of enteric bacilli (E. coli and Klebsiella spp.) were ESBL producer which is in congruous with previous study which recorded $19 \%$ of these organisms to be ESBL producer. Our study confirmed $22.2 \%$ E. coli as ESBL producer. This finding differed from previous studies which confirmed 27.2\% E. coli as ESBL producer (Khanna et al. 2013). The prevalence of ESBL producing Enterobacteriaceae varies greatly from country to country and among the hospitals within the country. Less than $1 \%$ to greater than $70 \%$ ESBLs is reported worldwide. The prevalence rates of ESBL in Nepal are reported frequently increasing from year to year.

\section{CONCLUSION}

The infection rates of indwelling devices used for various reasons are found to be very high. These infections have increased the morbidity and mortality of the hospitalized patients and also increased the duration of hospital stay.

\section{ACKNOWLEDGEMENTS}

We are grateful to KCMS family and all the staff members of Pathology Department of B \& B Hospital for their help and kind cooperation during laboratory work.

\section{CONFLICT OF INTEREST}

The authors declare no conflict of interest.

\section{REFERENCES}

Allen DM and Hartman BJ (2004). Acinetobacter species. In Bennett's principles and practice of infectious diseases. 6th ed. Churchill Livingstone, Philadelphia, pp. 2631-2635.

Amin NS, Shrestha ND and Bhat S (2013). Evaluation of semi quantitative culture method in the diagnosis of central venous intravascular catheter related infections. J Pharm Biomed Sci 26(26): 724-728.

Atela I, Coll P, Rello J, Quintana E, Barrio J, March F, Sanchez F, Barraquer P, Ballus J, Cotura A and Prats G (1997). Serial surveillance cultures of skin and catheter hub specimens from critically ill patients with central venous catheters: molecular epidemiology of infection and implications for clinical management and research. J Clin Microbiol 35(7): 1784-1790.

Bonten MJ, Slaughter S, Ambergen AW, Hayden MK, van Voorhis J, Nathan C and Weinstein RA (1998). The role of "colonization pressure" in the spread of vancomycin-resistant enterococci: an important infection control variable. Arch Intern Med 158: 1127-1132.

Deep A, Ghildiyal R, Kandian S and Shinkre N (2004). Clinical and Microbiological Profile of Nosocomial Infections in the Pediatric Intensive Care Unit (PICU). Indian Pediatrics 41: 1238-1246.

Dickinson GM and Bisno AL (1989). Infections associated with indwelling devices: infections related to extravascular devices. Antimicrob Agents Chemother 33: 602-607.

Downes KJ, Metlay JP, Bell LM, McGowan KL, Elliott MR and Shah SS (2008). Polymicrobial Bloodstream Infections among Children and Adolescents with Central Venous Catheters Evaluated in Ambulatory Care. Clinical Infectious Diseases 46(3): 387-94.

Elliott TS, Moss HA, Tebbs SE, Wilson IC, Bonser RS, Graham TR, Burke LP and Faroqui MH (1997). Novel approach to investigate a source of microbial contamination of central venous catheters. Eur J Clin Microbiol Infect Dis 16: 210213.

Gaynes R (2009). Definitions and epidemiology of nosocomial intravascular catheter-related (primary) bloodstream infections. Available: http:/ / www.uptodate.com. 
Isenberg HD (2004). Clinical Microbiology Procedure Handbook. 2nd Edition, ASM Press, Washington DC.

Jain P, Parada JP and David A (1995). Overuse of the indwelling urinary tract catheter in hospitalized medical patients. Arch Intern Med 155(13): 1425-1429.

Kalsoom F and Abdul H (2006). Resistance pattern of clinical isolates of Staphylococcus aureus against five groups of antibiotics, Multan. Pakistan J res Sci 17: 19-26.

Karlowsky JA, Jones ME, Draghi DC, Thornsberry C, Sahm DF and Volturo GA (2004). Prevalence and antimicrobial susceptibilities of bacteria isolated from blood cultures of hospitalized patients in the United States in 2002. Ann Clin Microbiol Antimicrob 3:1-8.

Khanna V,Mukhopadhayay C, K. E. V, Verma M and Dabke P (2013). Evaluation of central venous catheter associated blood stream infection: a microbiological observational study. Journal of pathogen 2-6.

Kunin CM, Douthitt S, Dancing J, Anderson J and Moeschberger M (1992). The association between the use of urinary catheters and morbidity and mortality among elderly patients in nursing homes. Am J Epidemiol 135: 291 - 301.

Mahto R, Amatya J, Amatya R and Adhikari D (2013). staphylococcal colonization in intravenous cannula and antimicrobial patterns of isolated Staphylococcus species. Nepal Journal of science and Technology 14(2): 131-136.

Maki DG, Stolz SM, Wheeler S and Mermel LA (1997). Prevention of central venous catheter-related bloodstream infection by use of an antisepticimpregnated catheter: a randomized, controlled trial, Ann Inter Med 127: 257-266.

Mermel LA (2000). Prevention of intravascular catheterrelated infections. Ann Intern Med 7; 132(5): 391-402.

O'Grady NP, Alexander M, Dellinger EP, Gerberding JL, Heard SO, Maki DG, Masur H, McCormick RD, Mermel LA, Pearson ML, Raad II, Randolph $A$ and Weinstein RA (2002). Guidelines for the prevention of intravascular catheter-related infections, Centers for Disease Control and Prevention. MMWR Recomm Rep. 9; 51(RR-10):129.

Parameswaran R, Sherchan JB, Varma D M, Mukhopadhyay C and Vidyasagar S (2011). Intravascular catheter-related infections in an
Indian tertiary care hospital. I Infect Dev Ctries 5(6): 452-458.

Peacock SJ, Eddleston M, Emptage A, King A and Crook DW (1998). Positive intravenous line tip cultures as predictors of bacteraemia. J Hosp Infect 40(1): 35-38.

Raad I, Hanna H and Maki D (2007). Intravascular catheter-related infections: advances in diagnosis, pre vention, and management. Lancet Infect Dis 7: 645-657.

Radji M, Fauziah S and Aribinuko N (2011). Antibiotic sensitivity pattern of bacterial pathogens in the intensive care unit of Fatmawati Hospital, Indonesia. Asian Pac J Trop Biomed 1(1): 39-42.

Rao SD, Joseph MP, Lavi R and Macaden R (2005). Infections related to vascular catheters in a pediatric intensive care unit. Indian Pediatrics 42(7): 667-672.

Riboli DFM, Lyra JC,Silva EP,Valadão LL, Bentlin MR,Corrente JE,de Souza Rugolo LMS andde Souza da Cunha MDLR (2014). Diagnostic accuracy of semi quantitative and quantitative culture techniques for the diagnosis of catheterrelated infections in newborns and molecular typing of isolated microorganisms. BMC Infectious Diseases 14: 283.

Sedor J and Mulholland SG (1999). Hospital-acquired urinary tract infections associated with theindwelling catheter. Urol Clin North Am 26(4): 821-828.

Stamm AM and Coutinho MS (1999). Urinary tract infection associated with indwelling bladder catheter: incidence and risk factors. Rev Assoc Med Bras 45(1): 27-33.

Storti A, Manzato AJ, Pizzolitto AC and Pizzolitto E (2006). Assessment of central venous catheterassociated infections using semiquantitative or quantitative culture methods. Journal of Basic and Applied Pharmaceutical Sciences 27(3): 213-220.

Tullu MS, Deshmukh CT and Baveja, SM (1998). Bacterial profile and antimicrobial susceptibility pattern in catheter related nosocomial infections. J Postgrad Med 44(1):7-13.

Xu Y, Moser C, Al-Soud WA, Sørensen S, Høiby N, Nielsen PH andThomsen TR (2012). CultureDependent and -Independent Investigations of Microbial Diversity on Urinary Catheters. J Clin Microbiol 50(12): 3901. 